\title{
REPRESENTATION OF MARKOV CHAINS BY RANDOM MAPS: EXISTENCE AND REGULARITY CONDITIONS
}

\author{
JÜRGEN JOST, MARTIN KELL, AND CHRISTIAN S. RODRIGUES
}

\begin{abstract}
We systematically investigate the problem of representing Markov chains by families of random maps, and which regularity of these maps can be achieved depending on the properties of the probability measures. Our key idea is to use techniques from optimal transport to select optimal such maps. Optimal transport theory also tells us how convexity properties of the supports of the measures translate into regularity properties of the maps via Legendre transforms. Thus, from this scheme, we cannot only deduce the representation by measurable random maps, but we can also obtain conditions for the representation by continuous random maps. Finally, we present conditions for the representation of Markov chain by random diffeomorphisms.
\end{abstract}

\section{INTRODUCTION}

Amongst the main concerns of Dynamics, one usually wants to decide whether asymptotic states of a given class of systems are robust under small random fluctuations. Such randomness, corresponding to natural fluctuations in physical processes, are represented by either a Markov chain model with localised transition or by a sequence of random maps. To see how they arise, consider a discrete-time system $f$ from a given topological space $M$ into itself. Suppose at each iteration of $f$ we allow a small mistake of size, say, at most $\varepsilon>0$. Then a Markov chain is defined by a family $\left\{p_{\varepsilon}(\cdot \mid x)\right\}$ of Borel probability measures, such that every $p_{\varepsilon}(\cdot \mid x)$ is supported inside the $\varepsilon$-neighbourhood of $f(x)$. The orbit of our dynamics subject to such small errors is thus given by sequences of random variables $\left\{x_{j}\right\}$, where each $x_{j+1}$ has distribution $p_{\varepsilon}\left(\cdot \mid x_{j}\right)$. Alternatively, one could think of the orbit as being made by the iteration $x_{j}=g_{j} \circ \cdots \circ g_{1}\left(x_{0}\right)$, where each measurable $g_{j}$ is picked at random $\varepsilon$-close, in a sense to be made more precise, from the original map $f$. Endowing the collection of maps $\left\{g_{j}\right\}$ with a probability distribution $\nu_{\varepsilon}$, we say that the sequence of random maps is a representation of that Markov chain if for every Borel subset $U$

$$
p_{\varepsilon}(U \mid x)=\nu_{\varepsilon}(\{g: g(x) \in U\}) .
$$

Date: September 10, 2018.

1991 Mathematics Subject Classification. 37H10, 37C05 (secondary), 37C40, 49K45, 49N60 (primary).

Key words and phrases. Markov chain, random dynamics, random maps, optimal transport, random diffeomorphisms, optimal coupling . 
In fact, given any sequence of random maps, one can always find a Markov chain which is represented by this scheme [BDV05, D.4], see also [ZaH07]; the Markov chain is simply given by (1), and one only needs to check that this satisfies the Markov chain criterion. The converse problem, however, is much more subtle, as we shall see. This is exactly the subject of this paper. In other words, we investigate under which conditions imposed on the Markov chain one can obtain a representation by a random map scheme and how its regularity properties are reflected.

The study of realisations of Markov chains via random maps goes back to Blumenthal and Corson [BlC70]. They considered the case where $M$ is a connected and locally connected compact metric space under some strong requirements on the probability measures. Let us denote by $\mathcal{P}(M)$ the space of all probability measures on $M$. In addition, suppose each $x \mapsto p(\cdot \mid x)$, acting from $M$ to $\mathcal{P}(M)$, is continuous relative to the weak* topology on $\mathcal{P}(M)$. Then, if for each $x$ the support of $p(\cdot \mid x)$ is all of $M$, they showed that it is possible to obtain a probability measure $\nu$ on the space of continuous transformations of $M$ such that condition (1) is fulfilled. Their proof is based upon the existence of a continuous projection of the subset of $\mathcal{P}(M)$ whose support is all of $M$ onto the space of probability measures on an interval whose support is the whole interval itself, and its continuous inverse. The assumption of full support on the probability measures is essential to assure the continuity of the maps. See for example [Kif86].

Weakening this condition, Kifer showed that if $M$ is a Borel subset of a complete separable metric space (Polish), then any Markov chain on $M$ can be represented by a sequence of measurable random maps [Kif86]. His idea was to use Borel measurable isomorphisms of $M$ to Borel subsets of the unity interval, as it had previously been shown by Kuratowski. Later, Quas [Qua91] tackled the case where $M$ is a smooth compact orientable Riemannian manifold. He showed that probability families which are absolutely continuous with respect to the normalised Riemannian measure whose density is smooth can be represented by $C^{\infty}$-random maps.

Afterwards, Araújo [Ara00] showed how to construct families of $C^{r}$-diffeomorphisms on the $n$-torus near an unperturbed $C^{r}$-diffeomorphism. He took advantage of the parallelisability of this manifold and of its quotient by integers. Then he used natural projections to identify orthonormal vector fields from which he could build these maps $C^{r}$-close to the original one; see [Ara00, Example 1]. Using a parametrised geodesic flow, he also showed the existence of parametrised families of diffeomorphisms, around an unperturbed one, of any compact boundaryless manifold; see [Ara00, Example 2]. Nevertheless, his procedures focus on uniformly continuous perturbations, requiring the small noise to uniformly cover a ball of positive radius around the unperturbed diffeomorphism. Furthermore, they do not yield a family of diffeomorphisms from a given probability distribution. 
More recently, Benedicks and Viana [BeV06, Example 1.7], and [BDV05, D.4] constructed random maps for small non-uniform noise. They lift implicitly the measure to the tangent space at $f(z)$, then try to transform the distribution to a fixed measure on $[0,1]^{n}$. If this transformation is invertible, its inverse regular enough, and varies smoothly with respect to $z$, then it is possible to select a random continuous map representing the perturbation. For topological reasons these constructions may fail on manifolds with nontrivial tangent bundle. Namely, their constructions implicitly assume the existence of global cross-section of the frame bundle.

In fact, it is not clear how to choose the random maps representing a Markov chain, and there might be many possible such choices. In such a situation, a basic strategy of geometric analysis is to select the maps according to some optimisation principle. This typically has the advantage that an object selected by an optimisation principle is not just some solution of the problem at hand; it typically enjoys additional properties derived from the optimisation, and these properties can typically be usefully exploited. This is also the strategy we adopt in the present paper. Since the maps should relate different measures, it is natural to select them by optimising a transportation problem between those measures. Thus, in this paper we introduce techniques from optimal transport in order to tackle the representation of Markov chains under different levels of regularity of the maps. The paper is organised as follows. After presenting the main definitions in Section 2, we review the main ingredients from optimal transport theory, in Section 3, to be used in the remaining part of the paper. In Section 4, we use optimal coupling to prove Theorem A, which shows how Markov chains can be represented by measurable maps. Then, in Section 5, we use Moser's coupling to show how representation by continuous random maps arise; the content of Theorem B. In the following Section 6, we discuss the regularity of densities and how they affect the properties of the transport maps. Then in the next Section 7, we use tangent bundle lifts of the measures and certain transformations to a fixed measure to construct continuous families of probabilities on the bundles. To tackle general manifolds, we rely on the fact that the tangent bundle of a manifold is always contained in a (smooth) trivial bundle, which can be seen by taking an isometric (Nash) embedding $M \hookrightarrow \mathbb{R}^{n}$. Then we lift (in a nice way) the measures from the tangent bundle to this trivial bundle to get a measure family $\left\{\mu_{x}\right\}_{x \in M}$ on $\mathbb{R}^{n}$. Using optimal transport theory and its regularity theory we get transformations to a fixed measure varying continuously with respect to $x \in M$ so that we can select sections of this bundle varying continuously, such that the distribution at a point $x$ represents the measure $\tilde{\mu}_{x}$. (Smooth) projections to the tangent bundle and the exponential map then give us the random continuous maps. These are summarised in Theorem C. Assuming further regularity, these maps are differentiable and we obtain random maps $C^{1}$-close to $f$, and if the unperturbed map $f$ is a diffeomorphism, we thus obtain a family of 
random diffeomorphisms. Therefore, we give a geometric condition for the representation of Markov chains by random diffeomorphisms.

In a subsequent paper, the methods developed here will be applied in order to address stochastic stability of several classes of dynamical systems. Starting from the seminal work of Kifer [Kif86, Kif88], we shall give conditions to stability in terms of Markov chains without the a priori assumptions of existence of random maps.

\section{Some notation And Definitions}

In this section we set up the notation and collect some main definitions to be used throughout this paper. For a comprehensive presentation on random perturbations of dynamics, see [Kif86, Kif88]. Although our main interest regards problems where the topological spaces under consideration are differential manifolds, some of the results that we will present are also true under lower requirements. We shall state it explicitly when that is the case. When we consider an arbitrary manifold though, say $M$, we suppose it to be compact and finite dimensional, equipped with some Riemannian structure, fixed once and for all, which induces a distance $d: M \times M \rightarrow \mathbb{R}$. We call $m$ its normalised Riemannian volume form on $M$, i.e. $m(M)=1$, and unless otherwise stated, we take absolute continuity with respect to $m$. As before, let us denote by $\mathcal{P}(M)$ the space of all Borel probability measures on $M$. As usual, $\mathcal{P}(M)$ is endowed with the weak* topology. The gradient operator will be denoted by $\nabla$, and the divergent by $\nabla \cdot$; the gradient of $f$ at the point $x$ will be denoted by $\nabla_{x} f$ or $\nabla f(x)$; the Laplace operator, i.e., the divergent of the gradient will be denoted by $\Delta$, and we use the same notation for $\mathbb{R}^{n}$ and for Riemannian manifolds.

Regarding measurability and continuity, we recall Lusin's theorem to be used in our proofs.

Theorem 2.1. [Fed69, Theorem 2.3.5]. Let $M$ be a locally compact metric space, $\mu$ a Borel measure on $M$, and $N$ a separable metric space. Let $f$ : $M \rightarrow N$ be a measurable map. Consider $A \subset M$ a measurable set with finite measure. Then for each $\delta>0$ there is a closed set $K \subset A$, with $\mu(A \backslash K)<\delta$ such that the restriction of $f$ to $K$ is continuous.

2.1. Markov chains and random maps. Let $N$ be separable complete metric space. We shall consider families of probability measures $\left(\mu_{x}\right)_{x \in M}$ in $\mathcal{P}(N)$ given by measurable maps $x \mapsto \mu_{x}$ with index set $M$. We speak of a continuous family of probability measures if the maps $x \mapsto \mu_{x}$ vary continuously from $M$ to $\mathcal{P}(N)$ relative to the weak* topology. In many cases one has $N=M$ or $N=\mathbb{R}^{n}$. Such families are sometimes called continuous Markov kernels. Therefore, Markov chains are special Markov kernels obeying some conditional probability with localised distribution.

Similarly, we can have a more general definition for our random maps. For an auxiliary probability space $(\Omega, \mathcal{A}, \mathbb{P})$, consider a measurable collection of maps $\mathcal{F}: \Omega \times M \rightarrow N,(\omega, x) \mapsto f_{\omega}(x)$. Then, we call the family 
$\left(f_{\omega}: M \rightarrow N\right)_{\omega \in \Omega}$ random measurable maps. If in addition each map in $\left(f_{\omega}: M \rightarrow N\right)_{\omega \in \Omega}$ is continuous, or a diffeomorphism, then we say that it is a family of random continuous maps, or random diffeomorphisms, respectively. They are also known as random fields. A representation of $\left(\mu_{x}\right)_{x \in M}$ is the mapping $\mathcal{F}: \Omega \times M \rightarrow N,(\omega, x) \mapsto f_{\omega}(x)$ such that for each $x$

$$
\mu_{x}=\mathcal{F}_{*} \mathbb{P} \text {. }
$$

Thus, we say that $\left(f_{\omega}\right)_{\omega \in \Omega}$ represents $\left(\mu_{x}\right)_{x \in M}$ if the distribution of $\omega \mapsto$ $f_{\omega}(x)$ equals $\mu_{x}$ for all $x \in M$.

\section{ON OPTIMAL TRANSPORT}

The remaining part of this paper is based upon techniques from optimal transport. Our main reference is the book by Villani [Vil09]. For the sake of completeness, we sample and collect in this section the concepts to be used along the way. Readers familiar with optimal transport may wish to skip this section and only refer back to it when needed.

The basic problem in optimal transport, as introduced by Monge, consisted in moving a given distribution like a pile of sand from one place to another with a minimal cost. There are several possible ways to generalise and tackle this problem. For example, the given mass to be transported can be thought of as a distribution in an appropriate probability space. In other words, given measurable spaces $M, N$, and probability measures $\mu$ in $\mathcal{P}(M)$ and $\nu$ in $\mathcal{P}(N)$, we seek for a coupling, or a way to connect these two measures. More generally, one has the following definition.

Definition 1. Let $(M, \mu)$ and $(N, \nu)$ be two probability spaces. We couple $\mu$ and $\nu$ by constructing two random variables $X, Y$ on some probability space $(\Omega, \mathbb{P})$, such that law $(X)=\mu$, law $(Y)=\nu$. The law or distribution of $(X, Y)$ is called coupling of $(\mu, \nu)$.

In our context, $\mu$ and $\nu$ are the only laws we shall be interested in, so we choose $\Omega=M \times N$. There are several examples of couplings arising in different contexts.

The first generalisation of Monge's original problem we can think of is given in terms of transport maps. That is, given measurable spaces $M, N$, probability measures $\mu$ in $\mathcal{P}(M)$ and $\nu$ in $\mathcal{P}(N)$, we seek for measurable maps $T: M \rightarrow N$, such that for all Borel $E \subset N$ one has $\mu\left(T^{-1}(E)\right)=\nu(E)$. This is an example of a so-called deterministic coupling. The requirement of a transport map, however, is a strong condition, and this problem may not have a solution unless more restrictions are made. The canonical example is when $\mu$ is a Dirac measure and $\nu$ is not.

In order to avoid ill-posed problems, one alternatively should look for weak solutions of the transport problem as it has been proposed by Kantorovich. In this case, we focus on probability measures $\gamma$ in $\mathcal{P}(M \times N)$, whose projections (or marginals) are $\mu$ and $\nu$. In other words, let $\Gamma(\mu, \nu) \subset$ $\mathcal{P}(M \times N)$, such that the canonical projections $\pi_{\mathcal{P}(M)}: \Gamma(\mu, \nu) \rightarrow \mathcal{P}(M)$ and 
$\pi_{\mathcal{P}(N)}: \Gamma(\mu, \nu) \rightarrow \mathcal{P}(N)$ hold. Then the marginals are given by the pushforward $\pi_{\mathcal{P}(M) *} \gamma=\mu$ and $\pi_{\mathcal{P}(N) *} \gamma=\nu$. The Kantorovich minimisation problem consists in obtaining

$$
C(\mu, \nu)=\inf _{\gamma \in \Gamma(\mu, \nu)} \int_{M \times N} c(x, y) d \gamma(x, y),
$$

where, for a given cost function $c: M \times N \rightarrow[0,+\infty]$, the infimum runs over the joint probabilities $\gamma$ in $\Gamma(\mu, \nu)$. The joint probability measures are called transport plans, the ones achieving the minimum, optimal transport plans, and $C(\mu, \nu)$ the optimal transport cost. Thus, this coupling is called optimal transport coupling. Obviously, the solution of the Kantorovich minimisation problem depends on the choice of the cost function. Although we state several of the auxiliary results from optimal transport in full generality, for our purpose we shall only use the quadradic cost function. The following theorem guarantees the existence of optimal coupling.

Theorem 3.1 (Existence of optimal coupling [Vil09, Theorem 4.1]). Given two Polish spaces $(M, \mu)$ and $(N, \nu)$, and a lower semicontinuous cost function bounded from below, then there always exist optimal couplings of $(\mu, \nu)$.

Notice that any transport map $T: M \rightarrow N$ induces a transfer plan $\gamma$ defined by $(I d \times T)_{*} \mu$. In fact, we can also impose conditions on the cost function such that the optimal coupling is obtained by a deterministic coupling. The search for a deterministic coupling (transport map) $T$ which minimises Eq. 3 for a given cost function is called the Monge problem. In other words, the Monge problem consists in finding deterministic optimal couplings realising

$$
\min \left\{\int_{M} c(x, T(x)) d \mu(x): T_{*} \mu=\nu\right\}
$$

$c: M \times N \rightarrow[0,+\infty]$. The following proposition ensures the existence of a unique transport map solving the Monge problem.

Proposition 3.2 (Solution of the Monge problem). Let $M$ be a Riemannian manifold, $\mathcal{X}$ a closed subset of $M$, with $\operatorname{dim}(\partial \mathcal{X}) \leq n-1$ and $\mathcal{Y}$ an arbitrary Polish space. Let $c: \mathcal{X} \times \mathcal{Y} \rightarrow \mathbb{R}$ be a continuous cost function, bounded from below and assume that for the probability measures $\mu \in \mathcal{P}(\mathcal{X})$ and $\nu \in \mathcal{P}(\mathcal{Y})$, the optimal cost $C(\mu, \nu)$ is finite. If the following conditions are fulfilled

i) $c$ is differentiable everywhere;

ii) $\mu$ is absolutely continuous;

iii) $\nabla_{x} c(x, \cdot)$ is injective where defined, i.e., if $x, y, y^{\prime}$ are such that $\nabla_{x} c(x, y)=$ $\nabla_{x} c\left(x, y^{\prime}\right)$, then $y=y^{\prime}$,

then there exists a unique (in law) optimal coupling $(x, y)$ of $(\mu, \nu)$, and it is deterministic.

Proof. The proof follows from Theorem 10.28, Proposition 10.7, and Remark 10.33 of [Vil09]. 
Corollary 3.3. Suppose that for each $k \in \mathbb{N}$ we have a sequence of continuous cost functions $c_{k}: \mathcal{X} \times \mathcal{Y} \rightarrow \mathbb{R}$ converging uniformly to $c: \mathcal{X} \times \mathcal{Y} \rightarrow \mathbb{R}$, where $c$ is defined as above. Let $\left(\nu_{k}\right)_{k \in \mathbb{N}}$ be a sequence of probabilities on $\mathcal{Y}$ converging weakly to $\nu \in \mathcal{P}(\mathcal{Y})$, and assume that for each $k$ there exist measurable maps $T_{k}: \mathcal{X} \rightarrow \mathcal{Y}$, such that each $T_{k}$ is an optimal transport map between $\mu$ and $\nu_{k}$. Then $T_{k}$ converges to $T$ in probability, i.e.,

$$
\forall \varepsilon>0 \quad \mu\left(\left\{x \in M ; d\left(T_{k}(x), T(x)\right) \geq \varepsilon\right\}\right) \underset{k \rightarrow \infty}{\longrightarrow} 0 .
$$

Proof. The proof follows from Proposition 3.2 above and [Vil09, Corollary 5.23].

Corollary 3.4. Let $M=\mathbb{R}^{n}$ and $c(x, y)=-x \cdot y$. Consider two probability measures $\mu, \nu$ on $M$, such that $\mu$ is absolutely continuous, then the solution of Monge's problem can be written as

$$
y=T(x)=x+\nabla \psi(x),
$$

where $\psi$ is some convex, lower semicontinuous function.

Proof. The proof follows from Theorem 10.44, Particular case 10.45, and Particular case 5.3 of [Vil09]. See also Section 6 below.

Remark 1. Proposition 3.2 is a slight variation of a more general theorem about the solution of the Monge problem. Its conditions can be weakened or replaced in a number of ways; see [Vil09, Theorem 10.28].

When the cost function is given in terms of distances in a metric space, we can use (3) in order to define a distance between measures.

Definition 2 (Wasserstein distances). Let $(M, d)$ be a Polish metric space, and $p \in[0, \infty)$. The Wasserstein distance of order $p$ between any two probability measures $\mu, \nu$ on $M$ is given by

$$
W_{p}(\mu, \nu)=\left(\inf _{\gamma \in \Gamma(\mu, \nu)} \int_{M} d(x, y)^{p} d \gamma(x, y)\right)^{1 / p} .
$$

Using the Wasserstein distances we can define a space given by the restriction on $\mathcal{P}(M) \times \mathcal{P}(M)$ on which $W_{p}$ takes finite values, or the space of probability measures with finite moment of order $p$.

Definition 3 (Wasserstein spaces). The Wasserstein space of order $p$ is given by

$$
\mathcal{P}_{p}(M)=\left\{\mu \in \mathcal{P}(M): \int_{M} d\left(x_{0}, x\right)^{p} \mu(d x)<\infty\right\} .
$$

The choice of $x_{0}$ is arbitrary and the space does not depend on this.

As a last important example we shall mention the powerful Moser coupling [Mos65, Vil09]. 
Theorem 3.5 (Moser coupling). Consider a smooth compact Riemannian manifold $M$ and its volume form $m$. In addition, consider Hölder continuous positive probability densities $\rho_{0}$ and $\rho_{1}$ on $M$. Then there is a deterministic coupling of $\mu_{0}=\rho_{0} m$ and $\mu_{1}=\rho_{1} m$. In other words, there exists a measurable map $T$ such that for all Borel $E \subset M$, we have $\mu_{1}(E)=T_{*} \mu_{0}(E)$. Furthermore, if $\rho_{0}, \rho_{1}$ are $C^{k, \alpha}$ then $T$ is $C^{k+1, \alpha}$.

The map $T$ is explicitly given, for each $x \in M$, in terms of a solution of the elliptic equation

$$
\Delta u(x)=\rho_{0}-\rho_{1},
$$

where $\Delta$ denotes the Laplace operator. The transport map is obtained by defining the locally Lipschitz vector field

$$
\xi(t, x)=\frac{\nabla u(x)}{(1-t) \rho_{0}(x)+t \rho_{1}(x)},
$$

which integrates to the flow $\left(T_{t}(x)\right)_{0 \leq t \leq 1}$ with an associated family of measures $\left(\mu_{t}\right)_{0<t<1}$. In particular, the time-1 map pushes forward $\mu_{0}$ to $\mu_{1}$. See [Vil09] for more details.

\section{Representation by meAsurable RANDOM MAPS}

From this section we begin to apply the techniques from optimal transport just presented. We shall start by tackling the problem of representing a Markov chain by measurable continuous maps. Our result implies Theorem 1.1 by Kifer [Kif86, Ch. 1]. We also treat the case of maps between different spaces, as introduced in Section 2.1. The main result of this section is

Theorem A. Let $M$ be a locally compact metric space and $N$ a locally compact Riemannian manifold. Consider a measurable family of probability measures $\left(\mu_{x}\right)_{x \in M}$ in $\mathcal{P}(N)$ with finite $p$-moments for $p \geq 1$, i.e. for some $y \in N$

$$
\sup _{x \in M} W_{p}\left(\delta_{y}, \mu_{x}\right)<\infty .
$$

Then there exist separable random measurable maps $\left(f_{\omega}: M \rightarrow N\right)_{\omega \in \Omega}$ representing $\left(\mu_{x}\right)_{x \in M}$.

Proof. Let $\nu$ be any probability measure absolutely continuous with respect to some volume measure on $N$, such that $W_{p}\left(\nu, \delta_{y}\right)<\infty$. Then Proposition 3.2 shows that for each $x \in M$ there is a unique optimal coupling realised by a (measurable) transport map $T_{x}: N \rightarrow N$, i.e.,

$$
W_{p}\left(\nu, \mu_{x}\right)^{p}=\int d\left(y, T_{x}(y)\right)^{p} d \nu(y) .
$$

Let $m$ be a Borel measure on $M$. By Lusin's theorem (Theorem 2.1), given the family $\left(\mu_{x}\right)_{x \in M}$ and $A \subset M$ with finite measure, for every $\delta>0$, there exists a set $K \subset A$, such that one has $m(A \backslash K)<\delta$ and the restriction of the measurable family of probability $x \mapsto \mu_{x}$ is continuous on $K$. Take any sequence $x_{k} \rightarrow x$ with $\left\{x, x_{1}, x_{2}, \ldots\right\} \subset K$. Then we have that $\mu_{x_{k}} \rightarrow \mu_{x}$, 
and Corollary 3.3 implies that $\nu\left(\left\{y \in N ; d\left(T_{x_{k}}(y), T_{x}(y) \geq \varepsilon\right\}\right) \rightarrow 0\right.$. Thus, these maps vary continuously in $x \in K$. Setting $(\Omega, \mathbb{P})=(\operatorname{supp} \nu, \nu)$, we can take for some random variable $Y: \Omega \rightarrow N$ with law $(Y)=\nu$ on $N$, and

$$
f_{\omega}(x):=T_{x}(Y(\omega)), x \in K, \omega \in \Omega .
$$

It follows that the maps satisfy (2), thus they represent the Markov chain. Moreover, they are measurable in $\omega$ and continuous in $x \in K$. Therefore, they are separable and jointly measurable on $\Omega \times K$. The sets $K$ such that this property holds generate a $\sigma$-algebra. Thus, there is a unique extension to the completion of the generated $\sigma$-algebra of $\Omega \times A$, and therefore, the random maps are separable and jointly measurable on $\Omega \times A$.

\section{REPRESENTATION BY CONTINUOUS RANDOM MAPS}

In this section we apply optimal transport and regularity theory to give conditions for the representation of Markov chains by continuous random maps. The main result of this section is the following theorem, which is closely related to the main result of Quas [Qua91].

Theorem B. Let $M$ be a complete separable metric space and $N$ a compact Riemannian manifold without boundary with normalised volume measure $m$. Assume $\left(\mu_{x}\right)_{x \in M}$ in $\mathcal{P}(N)$ to be a continuous family of probability measures, where each $\mu_{x}$ is absolutely continuous with respect to $m$ and has positive Hölder continuous (for some exponent $\alpha>0$ ) probability density varying continuously with $x \in M$ with respect to the $C^{0}$-topology.

Then $\left(\mu_{x}\right)_{x \in M}$ can be represented by random continuous maps $\left(f_{\omega}\right)_{\omega \in \Omega}$.

The proof is based on an application of Moser's coupling. We start with the following result.

Proposition 5.1. Let $M$ and $N$ be complete separable metric spaces and consider $\left(\mu_{x}\right)_{x \in M}$ in $\mathcal{P}(N)$ a continuous family of probability measures. Suppose for a fixed measure $\nu \in \mathcal{P}(N)$ with compact support there exists a family of continuous maps $\left(T_{x}: \operatorname{supp} \nu \rightarrow N\right)_{x \in M}$ varying continuously in the $C^{0}$ topology such that

$$
\left(T_{x}\right) \nu=\mu_{x} .
$$

Then $\left(\mu_{x}\right)_{x \in M}$ can be represented by random continuous maps $\left(f_{\omega}\right)_{\omega \in \Omega}$, such that $(\Omega, \mathbb{P})=(\operatorname{supp} \nu, \nu)$.

If, in addition, $T_{x}$ varies Hölder or Lipschitz continuously with respect to $x \in M$ then so does $f_{\omega}$ with the same constants (resp. exponents).

Proof. Set $(\Omega, \mathbb{P})=(\operatorname{supp} \nu, \nu)$ and let $X: \Omega \rightarrow \Omega$ be any random variable such that law $X=\nu$. Define

$$
f_{\omega}(x)=T_{x}(X(\omega)) .
$$

By construction, $\left(f_{\omega}\right)_{\omega \in \Omega}$ represents $\left(\mu_{x}\right)_{x \in M}$. We need to show that $f_{\omega}$ : $M \rightarrow N$ is continuous. Since $T_{x}$ varies continuously with respect to the 
$C^{0}$-topology and its domain is compact we have for a fixed $T_{x}$

$$
d\left(T_{x}(X(\omega)), T_{y}(X(\omega)) \leq d_{C^{0}}\left(T_{x}, T_{y}\right)<\epsilon\right.
$$

whenever $d(x, y)<\delta$ for sufficiently small $\delta=\delta(\epsilon, x)>0$.

Remark 2. Proposition 5.1 above generalises Example 1.7 by BenedicksViana [BeV06], where $\nu$ is the Lebesgue measure on $[0,1]^{n}$ and $T_{x}$ is the inverse of a rearrangement $S_{x}$ of the positive measure $\mu_{x} \in \mathcal{P}\left([-\epsilon, \epsilon]^{n}\right)$ to $[0,1]^{n}$, assuming $S_{x}$ is a homeomorphism which varies continuously in $C^{0}$.

In order to obtain random continuous maps, it is actually enough to assume that $x \mapsto T_{x}$ is pointwise continuous $\nu$-a.e. Then, if $x \rightarrow y$ for $\nu$-a.e. $\omega$ we have $T_{x}(\omega) \rightarrow T_{y}(\omega)$. We remark also that the assumption on the continuity of each $T_{x}$ could be relaxed by using Lusin's theorem. Furthermore, using a general version of the Kolmogorov-Chentsov continuity lemma for random fields obtained in [Pot09], a similar result holds if we only assume that each $T_{x}$ is Borel measurable converging fast enough as $x \rightarrow y$ in the topology of convergence in probability with respect to $(\Omega, \mathbb{P})$.

Proof of Theorem B. Let $\mu_{x}=\rho^{(x)} d m$. Then, by Theorem 3.5, there exists a coupling of $\left(m, \mu_{x}\right)$ induced by the time-1 map of a Lipschitz continuous vector field varying continuously with respect to $x \in M$. It is given by the solution of the following elliptic equation on $N$

$$
\Delta u^{(x)}=1-\rho^{(x)} .
$$

Since $\rho^{(x)}$ is $C^{0, \alpha}$, Schauder's theorem, see for example [Jos06, Ch. 11.2], implies that $u^{(x)}$ is $C^{2, \alpha}$. So we define the vector field on $N$

$$
\xi_{x}(t, y)=\frac{\nabla u^{(x)}(y)}{(1-t)+t \rho^{(x)}(y)} .
$$

According to our assumptions it is well-defined and integrates to a flow $T_{x, t}: N \rightarrow N$. Indeed, since $\rho$ is positive and $C^{0, \alpha}$, the vector field $\xi$ is likewise $C^{0, \alpha}$ w.r.t. $y$. Therefore, the flow is $C^{1, \alpha}$ w.r.t. $y$. Furthermore, we have

$$
\left(T_{x, 1}\right)_{*} m=\mu_{x}
$$

Notice that since the densities vary continuously with respect to the $C^{0}$ topology, so does the vector fields. This continuous dependence gives rise to a family of $T_{x, 1}$ also varying continuously with respect to the $C^{0}$-topology. Then, Proposition 5.1 implies that $\left(\mu_{x}\right)_{x \in M}$ can be represented by random continuous maps $\left(f_{\omega}\right)_{\omega \in \Omega}$.

\section{Regularity of Densities}

The next step is to establish conditions on representations of Markov chains by random diffeomorphisms. Before doing that, we shall again take up the discussion on optimal transport and its regularity properties applied to the regularity of the densities of Markov chains. We will focus on families of probabilities on $\mathbb{R}^{n}$. After that, we show how to use the results of this section 
on Riemannian manifolds via lifting and embedding techniques. We start with some technicalities, showing how convexity of the support of measures is related to the regularity of the transport maps.

Consider lower semi-continuous functions $\phi: U \subset \mathbb{R}^{n} \rightarrow \mathbb{R} \cup\{+\infty\}$, and $\psi: U^{\prime} \subset \mathbb{R}^{n} \rightarrow \mathbb{R}$, which will be hereafter called potentials. Then, let us define at $y \in U^{\prime} \subset \mathbb{R}^{n}$

$$
\phi^{c}(y)=\sup _{x \in U}(-c(x, y)-\phi(x)) .
$$

It is called the cost-transform or c-transform of $\phi$. Furthermore, let us define

$$
G_{\phi}(x)=\left\{y \in U^{\prime}: \phi(x)+\phi^{c}(y)=-c(x, y)\right\} .
$$

Then one can prove the following general result.

Theorem 6.1. Let $U, U^{\prime} \subset \mathbb{R}^{n}$ be bounded domains of $\mathbb{R}^{n}$, and $c(x, y)=$ $-x \cdot y$. Let $\mu$, and $\nu$ be probability measures on $U$, and $U^{\prime}$, respectively. Assume that $\mu$ does not give mass to sets of Hausdorff dimension less than or equal to $n-1$. Then there exist a $\mu$-a.e. unique $T$ solving the Monge problem for this cost function $c$. Moreover, there is a convex potential $\phi$ on $U$, such that $T=G_{\phi}$. Finally, if $\psi$ is convex and satisfies $\left(G_{\psi}\right)_{*} \mu=\nu$, then $\nabla \psi=\nabla \phi, \mu-a . e$.

See, for example, [Loe09, Theorem 2.7]. The condition of not giving mass to sets of Hausdorff dimension less than or equal to $n-1$ is satisfied if for some $p>n, \mu$ has $L^{p}$-density; see Proposition 3.3 of [Loe09]. In fact, for the cost function $c(x, y)=-x \cdot y$, one can show that $\phi=\psi^{c}$, where $\psi^{c}$ is the c-transform of $\psi$. In the case of this particular cost function, they are Legendre transforms of each other. See Particular case 5.3, and Definition 5.7 in [Vil09].

Regarding the regularity of $\phi$, one can prove the following result that we shall use in the sequel.

Theorem 6.2. Assume $c(x, y)=-x \cdot y$, and let $U, U^{\prime} \subset \mathbb{R}^{n}$ be bounded strictly convex. Suppose $\mu$ and $\nu$ are probability measures on $U$ and $V^{\prime} \subset U^{\prime}$, respectively, with $V^{\prime}$ being convex. Assume that for the convex potential $\phi$ and the cost function $c(x, y)$ we have $\left(G_{\phi}\right)_{*} \mu=\nu$. Furthermore, denoting the volume measure by $m$, assume that $\nu \geq \kappa m$ on $V^{\prime}$ for some $\kappa>0$, and $\mu$ satisfies for some $p \in] n, \infty]$ and $C_{\mu}>0$,

$$
\mu\left(B_{\varepsilon}(x)\right) \leq C_{\mu} \varepsilon^{n\left(1-\frac{1}{p}\right)} \quad \text { for all } \varepsilon>0 \text { and } x \in U
$$

Then $\phi$ is continuously differentiable on $U_{\delta}=\{x \in U \mid d(x, \partial U)>\delta\}$ with Hölder continuous derivatives for $\delta>0$. In particular, for some $\beta \in(0,1)$ and $\mathcal{C}$ depending only on $U, U^{\prime}, \kappa>0, \delta>0, p$ and $C_{\mu}$

$$
\|\phi\|_{C^{1, \beta}\left(U_{\delta}\right)} \leq \mathcal{C} \text {. }
$$

If, furthermore, $\mu$ is supported on some $\bar{V}$ compactly contained in $U$ then $G_{\phi}$ is a Hölder continuous map with Hölder norm bounded by $\mathcal{C}$. 
Proof. The proof directly follows from Theorem 3.4, Theorem 3.5 and Proposition 3.3 of [Loe09] using the particular chosen cost function $c(x, y)=$ $-x \cdot y$.

We remark that the integrability condition (6) is satisfied if, for example, for some $p>n, \mu$ has $L^{p}$-density. See Proposition 3.3 of [Loe09].

Consider some compact $K \subset \mathbb{R}^{n}$, and assume $\left(\mu_{x}\right)_{x \in K}$ to be a family of measures, such that each $\mu_{x}$ satisfies the assumption of $\mu$ in Theorem 6.2. Then, from Theorem 6.1 it follows that we can define optimal transport maps $S_{x}$ between $\mu_{x}$ and $\nu=m_{\mid[0,1]^{n}}$. Furthermore, from Theorem 6.2, these maps are Hölder continuous with Hölder norm bounded by a constant only depending on some compact convex neighbourhood $\Omega_{x}$ of the support of $\mu_{x}$. In particular, by uniqueness of the optimal transport map, we have the following Lemma.

Lemma 6.3. Let $\left(\mu_{x}\right)_{x \in K}$ be a continuous family of probability measures on some compact set $K \subset \mathbb{R}^{n}$. Suppose that each $\mu_{x}$ satisfies the assumptions of Theorem 6.2 with $C=C_{\mu_{x}}$ independent of $\mu_{x}$ and all supports contained in some convex $U \subset \mathbb{R}^{n}$. If we assume that the supports of $\mu_{x}$ are contained in $D=\operatorname{cl}\left(U_{\delta}\right)$ for some $\delta>0$ then the optimal transport maps $S_{x}$ between $\mu_{x}$ and $\nu=m_{\mid[0,1]^{n}}$ vary uniformly, that is if $x_{n} \rightarrow x$ then $S_{x}: D \rightarrow U^{\prime}$ varies continuously in the uniform topology of $C^{0}\left(D, U^{\prime}\right)$.

Proof. Because the $\|\cdot\|_{C^{1, \beta}}$-norm of $\left\{\phi_{x}\right\}_{x \in K}$ is uniformly bounded, the set is pre-compact in $C^{1}(D)$. Since up to a constant the potentials are unique we can assume $\phi_{x}\left(y_{0}\right)=0$ for all $x \in K$ and some $y_{0} \in D$. The limit of $\lim _{n \rightarrow \infty} \phi_{x_{n}} \rightarrow \tilde{\phi}_{x_{0}}$ for some $x_{n} \rightarrow x_{0}$ is also a convex potential solving the optimal transport problem with $\tilde{\phi}_{x_{0}}\left(y_{0}\right)=0$, which implies $\tilde{\phi}_{x_{0}}=\phi_{x_{0}}$. Therefore $\left\{\phi_{x}\right\}_{x \in K}$ is already closed and thus compact and $S_{x}: D \rightarrow U^{\prime}$ varies continuously in the $C^{0}$-topology as $x$ varies in $K$.

Proposition 6.4. Let $\left(\mu_{x}\right)_{x \in K} \subset \mathcal{P}\left(\mathbb{R}^{n}\right)$ be as above. In addition, assume each $\mu_{x}$ is supported on a convex set and has strictly positive Lebesgue density on its support, i.e. $\mu_{x} \geq \kappa \cdot m$ for some $\kappa>0$ independent of $x$, then

$$
S_{x \mid \operatorname{supp} \mu_{x}}: \operatorname{supp} \mu_{x} \rightarrow[0,1]^{n}
$$

is continuously invertible. Furthermore, if the supports vary continuously with respect to the Hausdorff metric on (compact) subsets of $\mathbb{R}^{n}$ then the inverse $T_{x}=S_{x}^{-1}:[0,1]^{n} \rightarrow \operatorname{supp} \mu_{x} \subset U$ varies continuously in the $C^{0}\left([0,1]^{n}, U\right)$. In addition, such a family $\left(\mu_{x}\right)_{x \in X}$ can be represented by random continuous maps.

Remark 3. If we do not assume that the supports vary continuously then it is still possible to show that the maps $T_{x}$ converge pointwise on $[0,1]^{n}$ to $T_{y}$ as $x$ converges to $y$ in $K$.

Proof. By Lemma $6.3, S_{x}$ varies continuously in $C^{0}\left(D, \mathbb{R}^{n}\right)$. Then Theorem 6.1 implies that the optimal transport problem from $\nu$ to $\mu_{x}$ has a 
unique continuous optimal transport map $T_{x}:[0,1]^{n} \rightarrow \operatorname{supp} \mu_{x}$ which, by Corollary 3.4, is the derivative of a differentiable convex potential $\psi_{x}$. Because $\psi_{x}$ is (up to a constant) the Legendre transform of the potential $\phi_{x}$, whose derivative is $S_{x}$, we necessarily have $T_{x}=S_{x}^{-1}$ on $[0,1]^{n}$.

To prove that $x \mapsto T_{x}$ is continuous it is sufficient to show that the graphs converge with respect to the Hausdorff metric. Because $x \mapsto S_{x}$ is continuous and the supports of $\mu_{x}$ vary continuously with respect to the Hausdorff metric, the restricted graph

$$
\widetilde{g r}\left(S_{x}\right)=\left\{\left(y, S_{x}(y)\right) \in \operatorname{supp} \mu_{x} \times[0,1]^{n}\right\}
$$

is continuous with respect to the Hausdorff metric on subsets of $\mathbb{R}^{n} \times \mathbb{R}^{n}$. But this implies

$$
x \mapsto \operatorname{gr}\left(T_{x}\right)=\left(\widetilde{g r}\left(S_{x}\right)\right)^{-1}
$$

is continuous and thus $x \mapsto T_{x}$ is continuous as well.

In particular, Proposition 5.1 implies that any such family $\left(\mu_{x}\right)_{x \in K}$ can be represented by random continuous maps.

Proposition 6.5. Suppose that $\mu$ is absolutely continuous with respect to the Lebesgue measure $m$ with $L^{p}$-density for some $p>n$. Furthermore, let $\nu=m_{\mid[0,1]^{n}}$. Then the transport map $S: \operatorname{supp} \mu \rightarrow[0,1]^{n}$ is continuously invertible on $U$, where $U$ is the set of point $z$ which admit a (convex) neighbourhood where the density of $\mu$ is strictly positive. In particular, this holds in $\{\rho>0\}=\operatorname{int}(\operatorname{supp} \mu)$ if the density $\rho$ of $\mu$ is continuous.

Proof. By Theorem 6.2 the map $S$ is (Hölder) continuous. Furthermore, the optimal transport map $T$ from $\nu$ to $\mu$ is almost everywhere the inverse of $S$ as they are the gradients of convex potentials $\phi$ and $\psi$, which are the Legendre transforms of each other. Thus, it suffices to show that $T$ is single-valued on $S(U)$. By definition we have

$$
S(x)=\{y \mid \phi(x)+\psi(y)=x \cdot y\}
$$

and

$$
\psi(y)=\sup \{x y-\phi(x)\},
$$

which implies that $S^{-1}(S(x))$ is convex. So if we show that $T$ is one-to-one on $S\left(V_{x}\right)$ for some small neighbourhood $V_{x}$ for all $x \in U$, then it implies that $S^{-1}(S(x)) \cap V_{x}=x$, i.e. $S$ and $T$ are both single-valued and thus continuous on resp. $U$ and $S(U)$.

Let $\pi$ be the optimal transport plan between $\nu$ and $\mu$, in particular we have

$$
(\mathrm{id} \times T)_{*} \nu=\pi
$$

Let $x$ be a point in $U$ and $V_{x}$ be a closed neighbourhood such that $\mu \geq \kappa m$ from some $\kappa>0$. By the restriction property for optimal transport plans [Vil09, Theorem 4.6] the plan $\tilde{\pi}_{\mid \mathbb{R}^{n} \times V_{x}}$ is an optimal transport plan between 
its marginal, i.e. between some $\tilde{\nu} \leq \nu$ and $\tilde{\mu}=\mu_{\mid V_{x}}$. Obviously this plan is also induced by $T$, i.e.

$$
\left(\text { id } \times T_{*}\right) \tilde{\nu}=\tilde{\pi} .
$$

Also note that $\tilde{\nu}$ is supported on $S\left(V_{x}\right)$.

The measures $\tilde{\nu}$ and $\tilde{\mu}$ satisfy the assumptions of Theorem 6.2, which implies that $T$ is (Hölder) continuous on $S\left(V_{x}\right)$ and hence one-to-one.

Corollary 6.6. Let $\mu$ and $\nu$ be as above. Assume the support of $\mu$ is strictly convex and

$$
U=\{\rho>0\}=\operatorname{int}(\operatorname{supp} \mu)
$$

where $\rho$ is the continuous density of $\mu$. Then the optimal transport map $T$ from $\nu$ to $\mu$ is a continuous map from $\operatorname{supp} \nu$ to $\operatorname{supp} \mu$.

Proof. Restrict $S$ to $\operatorname{supp} \mu$. By the previous theorem $S$ is injective in the interior of its domain. Furthermore, $S^{-1}(S(x))$ is convex. Because the support of $\mu$ is strictly convex this also implies $S$ is injective on the boundary, i.e. if $x^{\prime} \in S^{-1}(S(x))$ for $x \in \partial \operatorname{supp} \mu$ then $\lambda x^{\prime}+(1-\lambda) x \in \partial \operatorname{supp} \mu$ which implies $x=x^{\prime}$.

Because $S$ is one-to-one on its (convex) domain $\operatorname{supp} \mu$ and $S(\operatorname{supp} \mu)=$ $\operatorname{supp} \nu$, so is its inverse $T$ on $\operatorname{supp} \nu$. Thus, $T$ is continuous.

The two previous results show that one can only control the behaviour of the transport maps if the supports are strictly convex or the support convex and the density positive everywhere.

Proposition 6.7. Let $K$ be some (compact) set and $B_{1}$ be the closed unit ball in $\mathbb{R}^{n}$ and $\left(f_{x}: B_{1} \rightarrow f_{x}\left(B_{1}\right) \subset \mathbb{R}^{n}\right)_{x \in K}$ be a family of diffeomorphisms (onto their images) varying continuously in $C^{1}$ w.r.t. $x$. Assume $\left(\mu_{x}\right)_{x \in K}$ is a continuously varying family of measures supported on the images of $f_{x}$, i.e.

$$
\operatorname{supp} \mu_{x}=f_{x}\left(B_{1}\right)
$$

If each $\mu_{x}$ has $L^{\infty}$ (resp. continuous) density w.r.t. the Lebesgue measure then there is a (unique) continuously varying family $\left(\nu_{x}\right)_{x \in K}$ of measures with $L^{\infty}$ (resp. continuous) densities with $\left(f_{x}\right)_{*} \nu_{x}=\mu_{x}$.

Furthermore, if every point in the interior of the support of $\mu_{x}$ admits a neighbourhood such that the Lebesgue density of $\mu_{x}$ is strictly positive in the interior of the support then the same holds for $\nu_{x}$.

Proof. Assume $d \mu_{x}=\rho_{x} d m$ and there is some (Lebesgue regular) family $d \nu_{x}=\vartheta_{x} d m$ such that $\left(f_{x}\right)_{*} \nu_{x}=\mu_{x}$. By the Jacobian equations we have

$$
\vartheta_{x}(z)=\rho_{x}\left(f_{x}(z)\right) \cdot J_{f_{x}}(z) .
$$

So defining $\vartheta_{x}(z)$ as above gives us $\nu_{x}$.

Because $\left(f_{x}\right)_{x \in K}$ are continuously varying diffeomorphisms the Jacobians $J_{f_{x}}(z)$ vary continuously with respect to $(x, z) \in K \times B_{1}$ which implies the statement of the proposition. 
Corollary 6.8. Let $\left(\mu_{x}\right)_{x \in K}$ be as above. In addition, assume that each $\mu_{x}$ has continuous density which is strictly positive on the interior of its support. Then there exists a family of continuous maps $T_{x}:[0,1]^{n} \rightarrow \mathbb{R}^{n}$ varying in the $C^{0}$-topology and the following holds

$$
\left(T_{x}\right)_{*} m_{\mid[0,1]^{n}}=\mu_{x} .
$$

In particular, all such families $\left(\mu_{x}\right)_{x \in K}$ can be represented by random continuous maps $\left(f_{\omega}\right)_{\omega \in \Omega}$.

Proof. Just note the previous proposition implies that the family $\left(\nu_{x}\right)_{x \in K}$ and the measure $\nu=m_{\mid[0,1]^{n}}$ satisfies the assumptions of Corollary 6.6. Therefore, we can apply Proposition 6.4 to get continuous maps $\tilde{T}_{x}:[0,1]^{n} \rightarrow$ $\mathbb{R}^{n}$ varying continuously in the $C^{0}$-topology such that $\left(\tilde{T}_{x}\right)_{*} \nu=\nu_{x}$. Now it is easy to see that the maps $T_{x}=f_{x} \circ \tilde{T}_{x}$ satisfy the required assumptions. Similarly one gets a random continuous map $\left(g_{\omega}\right)_{\omega \in \Omega}$ representing $\left(\nu_{v}\right)_{x \in K}$. Then the random continuous map $\left(f_{x} \circ g_{\omega}\right)_{\omega \in \Omega}$ is representing $\left(\mu_{x}\right)_{x \in K}$.

Remark 4. The corollary can be applied if the supports are star-shaped with differentiably varying centre and radial function, i.e. there are $z_{x} \in B_{x}=$ $\operatorname{supp} \mu_{x}$ and differentiable maps

$$
r_{x}: \mathbb{S}^{n-1} \rightarrow(0, \infty)
$$

such that $x \mapsto\left(z_{x}, r_{x}\right)$ is continuous from $K$ to $\mathbb{R}^{n} \times C^{1}\left(\mathbb{S}^{n-1},(0, \infty)\right)$ (continuity of $x \mapsto z_{x}$ is enough to show that $J_{f_{x}}(z)$ is continuous in $\left.x\right)$.

The diffeomorphisms $f_{x}: B_{1} \rightarrow \mathbb{R}^{n}$ are constructed via

$$
f_{x}((\alpha, \rho))=z_{x}+\left(\alpha, r_{x}(\alpha) \cdot \rho\right) .
$$

(For simplicity we mixed polar coordinates $(\alpha, \rho)$ with Cartesian $z_{x}$ ).

\section{Measures on Bundles}

In the previous section we addressed the regularity of random maps depending on the properties of the family of measures. The constructions were almost entirely carried out on $\mathbb{R}^{n}$. In the next section, we shall tackle the problem of constructing random diffeomorphisms on Riemannian manifolds. In order to use the results on regularity, we shall lift the measures on the manifolds first to measures on their tangent bundles and then to measures on a trivial vector bundle containing the tangent bundle. The idea is to use the local equivalence via the exponential map of a neighbourhood of a point $p \in N$ and a neighbourhood of 0 of the tangent space at that point [Jos11] and that via Nash's embedding theorem the tangent bundle is contained in a trivial bundle. In this section we assume $M$ to be a complete separable metric space, and $N$ a locally compact Riemannian manifold. For $p \in N$, we shall locally endow $T_{p} N$ with the structure of a probability space via the exponential map $\exp _{p}: T_{p} N \rightarrow N$. We say that $Q \subset T_{p} N$ is measurable if $\exp _{p}(Q) \subset N$ is measurable. 
Given $\mu_{x} \in \mathcal{P}(N)$, a measure $\tilde{\mu}_{x} \in \mathcal{P}\left(T_{f(x)} N\right)$ can be defined as

$$
\tilde{\mu}_{x}=\left(\exp _{f(x)}^{-1}\right)_{*} \mu_{x}
$$

where $f: M \rightarrow N$ is some continuous map, and $f(x)$ belongs to the support of $\mu_{x}$. For example, it might be its centre of mass. In the case of randomly perturbed dynamics, in general $f$ is given by the unperturbed system if one considers (bounded) random perturbations. Thus, for $x \in M$, the mapping

$$
x \mapsto \mu_{x} \in \mathcal{P}(N),
$$

implicitly defines a mapping

$$
x \mapsto \tilde{\mu}_{x} \in \mathcal{P}\left(T_{f(x)} N\right) .
$$

Since the exponential map at a point $p \in N$ is a local diffeomorphism between a neighbourhood of that point and a neighbourhood of 0 in the tangent space at $p$, we obtain the following lemmata, whose proofs we leave to the reader.

Lemma 7.1. Let $M$ be a complete separable metric space, $N$ a Riemannian manifold. Consider a continuous family of probabilities $\left(\mu_{x}\right)_{x \in M}$ on $N$. Suppose that there is a $C^{r}$-map $f: M \rightarrow N$, for $r \geq 0$, such that for each $x$, the support of $\mu_{x}$ is contained in a sufficiently small neighbourhood $U_{f(x)}$ of $f(x)$.

Then $\left(\mu_{x}\right)_{x \in M}$ lifts to a continuous family of probability measures $\left(\tilde{\mu}_{x}\right)_{x \in M}$ on $T N$ with $\tilde{\mu}_{x}$ supported on $T_{f(x)} N$ (considered as a subset of $T N$ ).

Lemma 7.2. If, in addition to the assumptions above, $\mu_{x}$ is absolutely continuous with respect to a volume form on $N$, then $\tilde{\mu}_{x}$ is absolutely continuous with respect to the Lebesgue measure on $T_{f(x)} N$ (and by equivalence to the standard Lebesgue measure on $\mathbb{R}^{n}$ ).

Also, if $N$ is compact then the the Lebesgue densities of $\mu_{x}$ and $\tilde{\mu}_{x}$ are comparable in the sense that they have the same growth conditions, Lipschitzor Hölder-constants or positivity properties of the density in the interior of their support. And, in particular, (strict) convexity of the support of $\mu_{x}$ is preserved if the support is contained in a ball around $f(x)$ with radius less than the convexity radius of $N$.

If the tangent bundle is parallelisable, i.e.

$$
T N \cong N \times \mathbb{R}^{n},
$$

the mapping $x \mapsto \tilde{\mu}_{x}$ can be considered as a pair of maps

$$
x \mapsto\left(f(x), \tilde{\mu}_{x}\right) \in N \times \mathbb{R}^{n} .
$$

More generally, if there is a trivial bundle $F \cong N \times \mathbb{R}^{k}$ with a local equivalence $\rho: F \rightarrow N$ of a neighbourhood of 0 of $F_{f(x)}$ to the manifold (or the tangent space at $\left.T_{f(x)} N\right)$, then we can lift the measures via the local equivalence, by defining

$$
\hat{\mu}_{x}=\rho_{*} \mu_{x}
$$


Thus, for $x \in M$ we implicitly have the mapping

$$
x \mapsto \hat{\mu}_{x} \in \mathcal{P}\left(F_{f(x)}\right),
$$

and therefore the family $\left(\hat{\mu}_{x}\right)_{x \in M}$, which satisfies the assumptions that the original family $\left(\mu_{x}\right)_{x \in M}$ does.

In fact, one can always construct via isometric embedings a trivial bundle with a natural projection, and show that the measures on the manifolds can be lifted to such a bundle. As it is well know, Nash embedding theorem implies that the tangent bundle $T N$ of a $n$-dimensional manifold $N$ is a sub-bundle of the trivial bundle $F=N \times \mathbb{R}^{k}$ for some $k \geq n$ with a natural projection

$$
\pi: F \rightarrow T N
$$

which is a linear projection from $\mathbb{R}^{k}$ to $\mathbb{R}^{n}$ at each fibber at $p \in N$ [Jos11].

Suppose we have a fixed measure $\nu$ on $\mathbb{R}^{k}$ which has a smooth density with respect to the Lebesgue measure on $\mathbb{R}^{k}$, such that for any linear projection $r: \mathbb{R}^{k} \rightarrow \mathbb{R}^{n}$ the measure

$$
\nu^{(r)}=r_{*} \nu
$$

is absolutely continuous with respect to the Lebesgue measure on $\mathbb{R}^{n}$ with positive density inside the interior of its support. Then we claim that we can lift any Lebesgue regular measure $\tilde{\mu}$ on $\mathbb{R}^{n}$ with support in the interior of the support of $r_{*} \nu$ to a Lebesgue regular measure $\hat{\mu}$ on $\mathbb{R}^{k}$. To show this, notice that $\tilde{\mu}$ is absolutely continuous with respect to $\nu^{(r)}$, i.e.

$$
d \tilde{\mu}(x)=g(x) d \nu^{(r)}(x)
$$

then defining

$$
d \hat{\mu}(y)=g(r(y)) d \nu
$$

gives the required measure $\hat{\mu}$. Obviously, if the support of $\tilde{\mu}$ is convex so is the support of $\hat{\mu}$, as well as positivity in the interior is preserved by the lifts.

More generally, if there is a trivial bundle $F \cong N \times \mathbb{R}^{k}$ with a local equivalence $\rho: F \rightarrow N$ of a neighbourhood of 0 of $F_{f(x)}$ to the manifold (or the tangent bundle at $\left.T_{f(x)} N\right)$ such that we can lift the measures to

$$
x \mapsto \hat{\mu}_{x} \in \mathcal{P}\left(F_{f(x)}\right)
$$

with $\left(\hat{\mu}_{x}\right)_{x \in M}$ satisfying the assumptions of the previous section and

$$
\rho_{*} \hat{\mu}_{x}=\mu_{x}
$$

then there is a family of random maps representing $\left(\mu_{x}\right)_{x \in M}$.

\section{REPRESENTATION BY RANDOM MAPS WITH HIGHER REGULARITY}

In this section, we want to address the representation of Markov chains by random maps with higher regularity. In particular, our ultimate goal is to give conditions for the representation by random diffeomorphisms. Our first theorem in this section provides conditions for the representation by continuous random maps which we shall use thereafter to investigate the formal conditions for the representation by random diffeomorphisms. 
Before proceeding with, we present an example of the construction of random diffeomorphisms for the case when $M=N$ is parallelisable, based on [Ara00, Example 1].

Example 1. Let $M$ be any parallelisable $n$-dimensional $C^{k}$-Riemannian manifold, for $k \geq 1$. Consider $f: M \rightarrow M$, a $C^{r}$-diffeomorphism for $1 \leq r \leq$ $k$. We want to construct a family of random $C^{r}$-diffeomorphisms close to $f$ in the $C^{r}$-topology. Since $M$ is parallelisable, we have that $T M \cong M \times \mathbb{R}^{n}$, therefore there exists a globally orthonormal basis for the tangent space, that is, $n$ globally orthonormal vector fields $X_{1}(x)=(1,0, \ldots, 0), \ldots, X_{n}(x)=$ $(0,0, \ldots, 1)$ in $\mathfrak{X}^{r}(M)$ for all $x \in M$. Consider the probability space $\Omega=$ $[0,1]^{n}$. Then, for each $x \in M$, a family of random $C^{r}$-diffeomorphisms can be constructed as $f: \Omega \times M \rightarrow M$, where for $\omega=\left(\omega^{1}, \ldots, \omega^{n}\right) \in \Omega$ we have

$$
(\omega, x) \mapsto f(\omega, x)=f_{\omega}(x):=\exp _{f_{0}(x)}\left(\varepsilon \omega^{1} X_{1}\left(f_{0}(x)\right)+\cdots+\varepsilon \omega^{n} X_{n}\left(f_{0}(x)\right)\right),
$$

where we set $f_{0}(x):=f(x)$ for every $x \in M$. Notice that since $\omega$ is given by a certain distribution, not necessarily the uniform distribution on $[0,1]^{n}$, it induces a probability on the maps $f_{\omega}$. Taking $\varepsilon \rightarrow 0$ implies $\left\|f_{\omega}-f_{0}\right\|_{C^{r}} \rightarrow 0$. Recall that the space $\operatorname{Diff}^{r}(M)$ of diffeomorphisms is open in $C^{r}(M)$, for $r \geq 1$ [Hir76]. Thus, the perturbation we describe induces a probability on $\operatorname{Diff}^{r}(M)$, giving the random diffeomorphisms around $f_{0}$ with the same distribution of $\omega$ on the $C^{r}$-topology.

The situation is more complicated however for more general manifolds and perturbations. We start with the following result.

Theorem C. Let $M$ and $N$ be compact Riemannian $C^{k}$-manifolds without boundary, with $k \geq 1$. Let $m$ be the normalised volume measure on $N$. Consider $\left(\mu_{x}\right)_{x \in M}$, a continuous family of probability measures on $N$, such that each $\mu_{x}$ is absolutely continuous with respect to $m$, with positive continuous $L^{\infty}$-density $\rho_{x}$, and strictly convex support. Suppose that there is a $C^{r}$-diffeomorphism $f: M \rightarrow N$, for $r \leq k$, such that for each $x$, the support of $\mu_{x}$ is contained in a sufficiently small neighbourhood $U_{f(x)}$ of $f(x)$. Then $\left(\mu_{x}\right)_{x \in M}$ can be represented by a family $\left(f_{\omega}\right)_{\omega \in \Omega}$ of $C^{r}$-random continuous maps.

The proof of this theorem is based on regularity theory, the result on the conditions of representation of measures by continuous maps, and the lifting properties of measures that we have presented in the previous section. Indeed, as discussed in Section 7, it is possible to lift the measures on $N$ to its tangent bundle via the exponential map and we can identify $T N$ with a sub-bundle of a trivial bundle $N \times \mathbb{R}^{l}$ [Jos11]. Then Lemma 7.1, and Lemma 7.2 provide a natural way of constructing a continuous family of probabilities on the bundles according to $\left(\mu_{x}\right)_{x \in M}$ on $N$. Furthermore, the construction of a trivial bundle shows a natural way of embedding, thus lifting again this measures to some $\mathbb{R}^{k}$, such that the results of Section 6 can be applied. In other words, we can continuously select sections of the bundles. Thus, we choose maps $C^{r}$-close to $f$ according to $\mu_{x}$. 
Proof of Theorem C. We divide the proof into two cases. Namely, when the manifold $N$ has trivial bundle, and when it has not.

Case I: Parallelisable manifolds: Let us begin with the case when $N$ has a trivial bundle. We start with the following.

Step 1: There exists a continuous family $\left(\tilde{\mu}_{x}\right)_{x \in M}$ on $T N$ and open neighbourhoods $V_{f(x)} \subset T_{f(x)} N$, such that every supp $\tilde{\mu}_{x} \subset V_{f(x)}$. Indeed, since by hypothesis the support of each $\mu_{x}$ is contained in some small open $U_{f(x)}$, from Lemma 7.1 , the family $\left(\mu_{x}\right)_{x \in M}$ is lifted to a continuous family $\left(\tilde{\mu}_{x}\right)_{x \in M}$ on $T N$. Furthermore, since the probabilities are lifted via the exponential map, each $\tilde{\mu}_{x}$ is supported in small neighbourhoods $V_{f(x)} \subset T_{f(x)} N \subset T N$. Step 2: Each $\tilde{\mu}_{x}$ has strictly convex support and is absolutely continuous with respect to the volume measure on $T N$, with densities $\gamma_{x}$ as regular as $\rho_{x}$. Indeed, Lemma 7.2 gives us the regularity conditions and assures us the strictly positive density on the interior of the support of each $\tilde{\mu}_{x}$. Furthermore, it shows that the support of each $\tilde{\mu}_{x}$ is strictly convex.

Step 3: The family $\left(\tilde{\mu}_{x}\right)_{x \in M}$ can be represented by random continuous maps. Note that $\tilde{\mu}_{x}=\gamma_{x} \mathrm{Vol}$, and that the density $\gamma_{x}$ can be written as $\gamma_{x}=\exp _{f(x)}^{-1} \circ \rho_{x}$. Since $\rho_{x}$ is $L^{\infty}(m)$, and the exponential map is locally a $C^{\infty}$-diffeomorphism, we have that over bounded domains each measure on the bundle also has bounded densities, i.e., $\gamma_{x}$ is $L^{\infty}(\mathrm{Vol})$. Furthermore, parallelisability of $T N$ implies that $T N \cong N \times \mathbb{R}^{n}$. In particular, we have $\operatorname{supp} \tilde{\mu}_{x} \supset f(x) \times \mathbb{R}^{n}$. Therefore, we can assume that each $\tilde{\mu}_{x}$ lives on the same $\mathbb{R}^{n}$. Now the continuous family of probability that we have just constructed fulfils the conditions of Corollary 6.8. Thus, they can be represented by a random continuous map $\left(\tilde{f}_{\omega}: M \rightarrow T N\right)_{\omega \in \Omega}$.

Step 4: The family $\left(\mu_{x}\right)_{x \in M}$ by random continuous maps. Using the exponential map exp $: T N \rightarrow N$, set $f_{\omega}:=\exp \circ \tilde{f}_{\omega}$. By construction of the measure family $\left(\tilde{\mu}_{x}\right)_{x \in M}$ one can easily verify that $\left(f_{\omega}\right)_{\omega \in \Omega}$ represents $\left(\mu_{x}\right)_{x \in M}$.

Case II: General manifolds In this case note that $T N$ is contained in a trivial vector bundle $N \times \mathbb{R}^{k}$ such that the natural embedding $e: T N \rightarrow N \times \mathbb{R}^{k}$ is smooth.

Step 1' There exists a continuous family $\left(\tilde{\mu}_{x}\right)_{x \in M}$ on $N \times \mathbb{R}^{k}$ and open neighbourhoods $V_{f(x)} \subset f(x) \times \mathbb{R}^{k}$, such that every $\operatorname{supp} \tilde{\mu}_{x} \subset V_{f(x)}$. Just note that as above one can first lift the measures $\mu_{x}$ to a family $\hat{\mu}_{x}$ living on $T N$. By assumption, each measure has density w.r.t. the Lebesgue measure on $T_{f(x)} N$. As the embedding $e$ is smooth, if take the Lebesgue measure on $\mathbb{R}^{k} \equiv f(x) \times \mathbb{R}^{k}$, restrict it to a sufficiently small ball $\lambda_{\mid B_{\epsilon}(0)}^{k}$, then the pushforward has density w.r.t. the Lebesgue measure on $T_{f(x)} N$ which is smooth in the interior of a small ball $B_{\delta} \subset T_{f(x)} N$. By duality, we can pull-back the densities of the measures $\hat{\mu}_{x}$ to get measures $\tilde{\mu}_{x}$ with Lebesgue density. Steps 2' and 3' As steps 2 and 3 above. 
Step 4' The family $\left(\mu_{x}\right)_{x \in M}$ by random continuous maps. As we obtained a random continuous map $\left(\tilde{f}_{\omega}: M \rightarrow N \times \mathbb{R}^{k}\right)$, we only need to define $f_{\omega}:=e \circ \exp \circ \tilde{f}_{\omega}$ to obtain the required family.

8.1. Conditions for a representation by random diffeomorphisms. The proof of Theorem $\mathrm{C}$ showed that a crucial step was the construction of random continuous maps $\left(f_{\omega}: M \rightarrow \mathbb{R}^{n}\right)$. As the (sufficiently regular) solutions of the optimal transport problem solve an elliptic partial differential equation, namely the Monge-Ampère equation, whose boundary conditions are given in terms of densities, we can get regularity conditions from general principles of elliptic regularity theory. Formally, this works as follows. Let $F: \Lambda \times C(N) \rightarrow \mathbb{R}$ be the solution operator of an elliptic equation on $N$ depending on the parameter $x \in \Lambda$, for some parameter space $\Lambda$. In our case, $\Lambda$ will stand for boundary values, and in fact, we have $\Lambda=M$. We then have

$$
0=\frac{d}{d x} F=\frac{\partial F}{\partial x}+\frac{\partial F}{\partial \varphi} \cdot \frac{d \varphi}{d x}
$$

In the elliptic case, for each $x$, the solution $\varphi$ is unique and satisfies a-priori estimates, that is, it is controlled by the data of the equation $F$. This means that we can control $\frac{\partial \varphi}{\partial F}$. Therefore, the derivative $\frac{\partial F}{\partial \varphi}$ is invertible, and we obtain

$$
\frac{d \varphi}{d x}=\left(\frac{\partial F}{\partial \varphi}\right)^{-1} \frac{\partial F}{\partial x} .
$$

A similar formal calculation works for higher order derivatives.

In our situation, the elliptic equation is the Monge-Ampère equation, i.e. $F\left(x, \varphi_{x}\right)=0$, if and only if, $\varphi_{x}$ solves the Monge-Ampère equation under the (second boundary) condition $\left(\nabla \varphi_{x}\right)_{*} \mu_{0}=\mu_{x}$, for which the regularity theory is developed in [MTW05, TW09].

Thus, for our purposes, we only need to explicitly verify the dependence of $F$ on $x$ and the ellipticity of the boundary value problem, that is, apriori estimates for a solution $\varphi$ of the Monge-Ampère equation under the boundary condition $(\nabla \varphi)_{*} \mu_{0}=\mu$. These have been obtained in [TW09, Theorem 1.1]. More precisely, for the case of a quadratic cost function as considered here, that result yields a $C^{2}$-estimate for $\varphi$ in terms of the geometry of $N, \mu_{0}, \mu$ and $\sup |\varphi|$, and this estimate implies uniqueness, see [TW09, Theorem 1.2]. From this, one may obtain $C^{2, \alpha}$-estimates, and linear elliptic regularity theory then yields higher order estimates in a standard manner. Then the above formula yields the dependence of the solution $\varphi_{x}$ on the parameter $x$. That is, a smooth dependence of $\mu_{x}$ on $x \in M$ will translate into a corresponding smooth dependence of $\varphi_{x}$ on $x$. In particular, the transport maps will vary smoothly. In addition, we have the following proposition.

Proposition 8.1. Assume that ellipticity conditions as discussed above are satisfied so that the solution of the Monge-Ampère equation varies smoothly 
w.r.t. the parameter $x \in M$. If $f: M \rightarrow N$ is a diffeomorphism as in Theorem $C$ and the perturbation is sufficiently small, then the constructed random continuous map from Theorem $C$ is in fact a random diffeomorphism.

Proof. Just note that the random map is constructed via an exchange of parameter, i.e.

$$
f_{\omega}(x)=T_{x}(\omega)
$$

where $\omega \in \Omega=B_{1}(0) \subset \mathbb{R}^{n}$. The condition of smooth dependence on $x \in M$ is equivalent to saying that each $f_{\omega}$ is smooth (uniformly) dependent on $x \in M$. Therefore, if the perturbation is sufficiently small, then the maps converge on the $C^{r}$-norm to the unperturbed map $f$. As the set of diffeomorphisms $\operatorname{Diff}(M, N)$ is open in $C^{r}(M, N)$, we have that $f_{\omega}$ must be a diffeomorphism as well.

Therefore, Proposition 8.1 and the discussion right before it tell us that if $\mu_{x}$ fulfils the conditions in Theorem $\mathrm{C}$ and depends smoothly on $x \in M$, it can be represented by random diffeomorphisms.

\section{ACKNowledgements}

The research leading to these results has received funding from the European Research Council under the European Union's Seventh Framework Programme (FP7/2007-2013) / ERC grant agreement $n^{\circ}$ 267087. M.K. was supported by the International Max Planck Research School "Mathematics in the Sciences". We would like to thank the anonymous referee for the careful reading and constructive comments, which have contributed to substantially improve the presentation of this manuscript.

\section{REFERENCES}

[Ara00] V. Araújo, Attractors and time averages for random maps., Annales de l'Institut Henri Poincaré Analyse Non Linéaire 17 (2000), no. 3, 307-369.

[BeV06] M. Benedicks and M. Viana, Random perturbations and statistical properties of Hénon-like maps., Annales de l'Institut Henri Poincaré Analyse Non Linéaire 23 (2006), no. 5, 713-752.

[BlC70] R. M. Blumenthal and H. H. Corson, On continuous collections of measures., Annales de l'Institut Fourier 20 (1970), no. 2, 193-199.

[BDV05] C. Bonatti, L. J. Díaz, and M. Viana, Dynamics beyond uniform hyperbolicity: a global geometric and probabilistic perspective, Encyclopedia of Mathematical Sciences, vol 102, Springer, Berlin, 2005.

[Fed69] H. Federer, Geometric measure theory, Grundlehren der Mathematischen Wissenschaften, vol. 153, Springer, New York, 1969.

[Hir76] M. W. Hirsch, Differential topology, Springer-Verlag, Berlin, 1976.

[Jos06] J. Jost, Partial Differential Equations, Springer, Berlin, 2006.

[Jos11] J. Jost, Riemannian Geometry and Geometric Analysis, Springer, Berlin, 2011.

[Kif86] Yu. Kifer, Ergodic theory of random transformations., Birkhäuser, Boston, 1986.

[Kif88] Yu. Kifer, Random perturbations of dynamical systems., Birkhäuser, Boston, 1988. 
[Loe09] G. Loeper, On the regularity of solutions of optimal transportation problems, Acta Mathematica 202 (2009), no. 2, 241-283.

[MTW05] X.N.Ma, N.Trudinger and X-J.Wang, Regularity of potential functions of the optimal transportation problem, Arch. Rat. Mech. Anal.177(2005), 151-183.

[Mos65] J. Moser, On the volume elements on a manifold, Trans. Amer. Math. Soc., 120 (1965), 286-294.

[Pot09] J. Potthoff, Sample properties o random fields II: continuity, Communications in stochastic analysis, 3 (2009), no. 3, 331-348.

[Qua91] A. N. Quas, On representation of Markov chains by random smooth maps, Bulletin of the London Mathematical Society 23 (1991), no. 5, 487-492.

[TW09] N.Trudinger and X.J.Wang, On the second boundary value problem for MongeAmpère type equations and optimal transportation, Ann. Scuola Norm. Sup. Pisa Cl. Sci. 8 (2009), 143174.

[Vil09] C. Villani, Optimal transport: old and new, Grundlehren der Mathematischen Wissenschaften, vol. 338, Springer, Berlin, 2009.

[ZaH07] H. Zmarrou and A.J. Homburg, Bifurcations of stationary measures of random diffeomorphisms, Ergodic Theory and Dynamical Systems 27 (2007), no. 5, 1651-1692.

Jürgen Jost, MaX-Planck-Institute for Mathematics in the Sciences, InselStr. 22, 04103 Leipzig, Germany, and Department of Mathematics, University of Leipzig, 04081 Leipzig, Germany

E-mail address: jost@mis.mpg.de

Martin Kell, MaX-Planck-Institute for Mathematics in the Sciences, InSElSTR. 22, 04103 LEIPZIG, Germany

E-mail address: mkell@mis.mpg.de

Christian S. Rodrigues, Max-Planck-Institute for Mathematics in the Sciences, Inselstr. 22, 04103 Leipzig, Germany

E-mail address: christian.rodrigues@mis.mpg.de 